\title{
LINGUISTIC ACCULTURATION AND PERCEPTIONS OF QUALITY, ACCESS, AND DISCRIMINATION IN THE HEALTH CARE AMONG LATINOS IN THE UNITED STATES
}

\section{David Becerra | David Androff | Jill T. Messing | Jason Castillo | Andrea Cimino}

\section{Latinos in the United States}

The Latino population is the largest and among fastest growing minority populations in the United States (US) (Humes, Jones \& Ramirez, 2011). However, previous research shows that Latinos, especially those of Mexican heritage and monolingual Spanish speakers, underutilize health care services (Fiscella, Franks, Doescher, \& Saver, 2002; Vargas Bustamante, Fang, Rizzo, \& Ortega, 2009).

\section{Purpose of the Study}

This research examined the relationship between linguistic acculturation and Latinos' perceptions of quality of medical care, access to medical information, the ability to fill out medical forms, and discrimination.

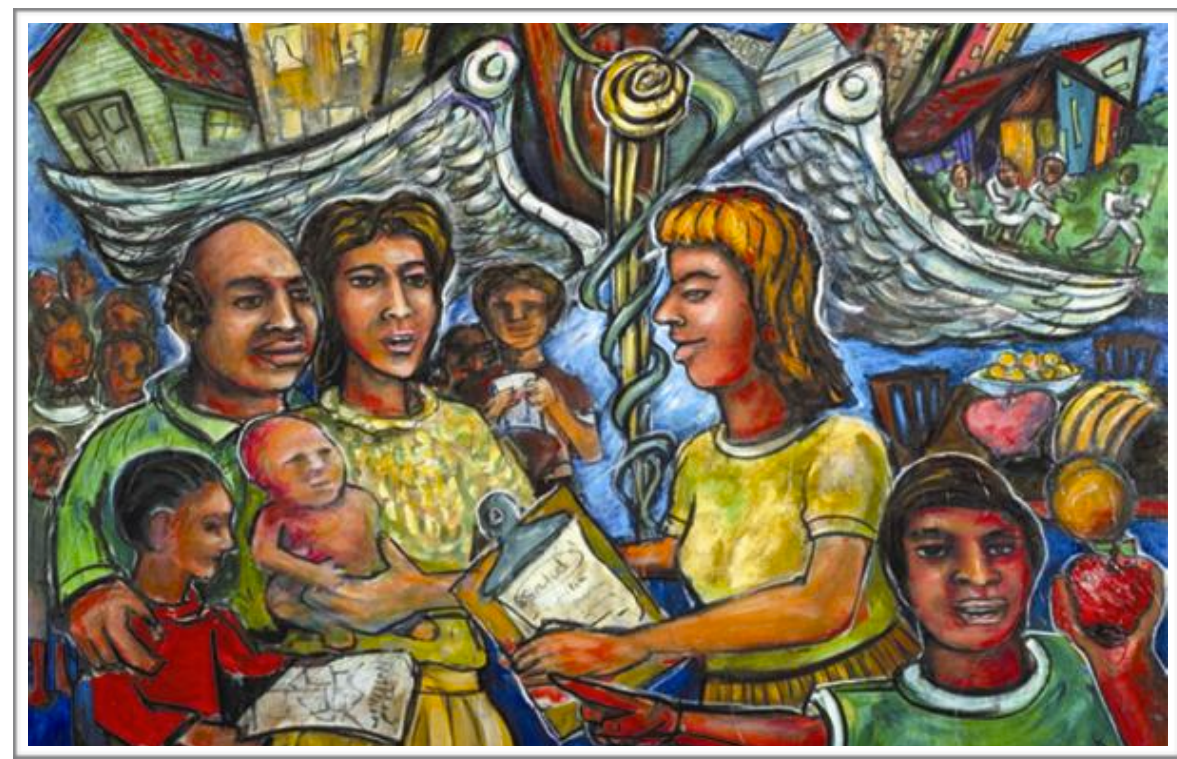

Source: (Xavier Cortada, 2003)

The Latino population is the largest and among fastest growing minority populations in the United States (US) (Humes, Jones \& Ramirez, 20II). However, previous research shows that Latinos, especially those of Mexican heritage and monolingual Spanish speakers, underutilize health care services (Fiscella, Franks, Doescher, \& Saver, 2002; Vargas Bustamante, Fang, Rizzo, \& Ortega, 2009). Factors that appear to negatively impact Latinos' utilization of health care services include sex, age, socioeconomic status, level of acculturation (Trivendi \& Ayania, 2006), and perceived discrimination by health care providers on account of their race and/or ethnicity (Perez, Sribney, \& Rodriguez, 2009). While it would seem that health insurance coverage would be a predictor of health care access and health care seeking behaviors, health coverage does not guarantee high quality health care or non-discrimination in health

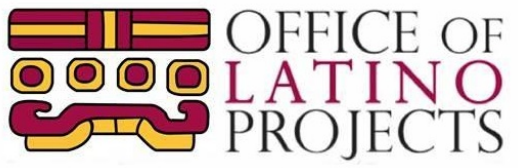
care settings. 


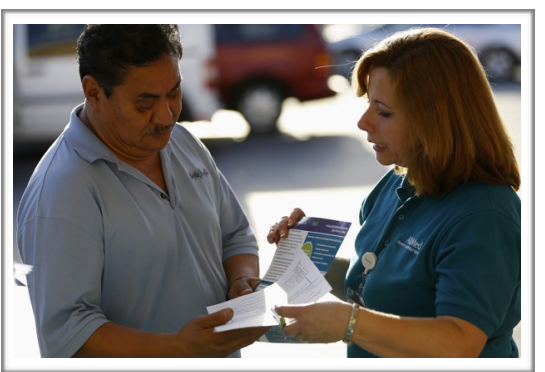

Source: (Mario Azuoni/Reuters)

\section{Latino Paradox}

Latinos living in the US often

have low socioeconomic status, low levels of education, and lower levels of health insurance coverage, which can be greater risk factors for poor health; however, those factors do not always lead to negative health outcomes among many Latinos. This phenomenon-known as the Latino Paradox-indicates that Latinos tend to be healthier than other racial ethnic groups with similar socioeconomic status (Franzini \& FernandezEsquer, 2004).

\section{Latino Culture}

Latinos adhere strongly to traditional Latino culture, and this adherence has been found to protect Latinos against negative health risks (Masiglia, Kulis, Martinez-Rodriguez, Becerra, \& Castillo, 2009; PérezEscamilla, 2011).
Patients rely heavily on doctors' ability to accurately diagnose them. Research suggests that approximately 12 million adults are misdiagnosed on an annual basis. In addition, it is estimated that errors occur in up to II \% of prescriptions (Sandars \& Esmail, 2003). For an accurate diagnosis, a mutual exchange of information is required; doctors must listen and gather information during the patient's medical interview or assessment. Patients must then make informed decisions based on the information provided by doctors. But previous research demonstrates that while health care providers have made attempts to increase cultural competence, patients from diverse racial, ethnic, and linguistic backgrounds often report inadequate attention and empathy from their White doctors (Peck \& Denney, 20I2; Perloff, Bonder, Ray, Ray, \& Siminoff, 2006; Pilnick \& Dingwall, 20II). This phenomenon affects the ability of doctors to diagnose and treat patients, which is detrimental to patient satisfaction and treatment adherence (Ferguson \& Candib, 2OO2). The doctor-patient relationship is further affected by a patient's inability to understand medical information, accurately complete medical forms and their perceptions of discrimination.

Latinos living in the US often have low socioeconomic status, low levels of education, and lower levels of health insurance coverage, which can be greater risk factors for poor health. However, among many Latino immigrants, those factors do not always lead to negative health outcomes. This phenomenon - known as the Latino Paradox - indicates that Latinos tend to be healthier than other racial ethnic groups with similar socioeconomic status (Franzini \& Fernandez-Esquer, 2004). Latinos adhere strongly to traditional Latino culture, and this adherence has been found to protect Latinos against negative health risks (Masiglia, Kulis, Martinez-Rodriguez, Becerra, \& Castillo, 2009; Pérez-Escamilla, 20II). However, these protective factors associated with Latino heritage often diminish with the time, and various stressors - such as immigration status, separation from families, poverty, low levels of education, lack of job skills, English language difficulties, and experiences of prejudice and discrimination - can place Latinos at greater risk for negative health outcomes (Alderete, Vega, Kolody, \& Aguilar-Gaxiola, 2000; CavazosRehg, Zayas, \& Spitznagel, 2007; Pumariega, Rothe, \& Pumariega, 2005).

These protective factors also diminish through acculturation; the process by which immigrants adopt the customs, language, food, and societal norms of the host country. Acculturation has been found to cause considerable stress among Latinos who immigrate to the United States (Kouyoumdjian, Zamboanga, \& Hansen, 2003). One important indicator of acculturation is an individual's English language use and fluency, referred to as linguistic acculturation. Studies suggest there is an association between linguistic acculturation and utilization of health care services. For example, a study of over 13,000 persons found that speaking primarily Spanish versus English, at home, was linked with not seeking recommended health care services (Cheng, Chen, \& Cunningham's, 2007). Language barriers and discrimination in health care settings, make it difficult for Spanish-dominant speakers to access care and impact the satisfaction received from health services (Huang, Appel, \& Ai, 20II). The same perceived discrimination can in turn exacerbate the stress felt by Latino immigrants and contribute to additional mental health issues (Ayón \& Becerra, 2013; Gonzalez, Fabrett, \& Knight, 2009; Umaña-Taylor \& Alfaro, 2009). 


\section{Study purpose}

As previously mentioned, the US Spanish-speaking Latino population continues to increase, making necessary to further examine Latinos' perceptions of quality, access, and discrimination in healthcare treatment. This research ${ }^{1}$ examined the relationship between linguistic acculturation and Latinos' perceptions of quality of medical care, access to medical information, the ability to fill out medical forms, and discrimination. It was hypothesized that less linguistically acculturated participants would report: (I) lower quality of health care treatment; (2) lower confidence filling out medical forms; and (3) greater difficulty understanding written medical information. It was also hypothesized that participants who report having received poor quality medical treatment, were less linguistically acculturated, and were more likely to report that their poor treatment was a result of (4) an inability to pay; (5) their racial/ethnic background; and (6) their accent.

\section{Methodology}

The sample consisted of 4 , OI 3 participant's ages i 8 and older. Of those, 2,783 (69.5\%) were foreign-born and I,220 (30.5\%) were U.S.-born. The sample consisted of $2,016(50.2 \%)$ males and I,997 (49.8\%) females, whose ages ranged from 18 to 92. The interviews were conducted by phone, in both English and Spanish, utilizing disproportionate stratified random digit dialing (RDD). Among the participants $25.9 \%$ preferred to be interviewed in English and 72.I\% preferred to be interviewed in Spanish. A grand majority of participants (90.2\%) reported receiving their health care from a doctor's office, community health clinic or other outpatient clinics.

The variable linguistic acculturation consisted of a three-item scale. ${ }^{2}$ The scores on this scale were reverse coded, ranging from three to ten, so that the higher scores indicated lower levels of linguistic acculturation. The mean score for linguistic acculturation scale was $6.55(\mathrm{SD}=2.57)$. Further, to examine perceptions about issues of quality discrimination in their medical care, and access to medical information, participants were asked a series of questions ${ }^{3}$ and measured with Likert scales.

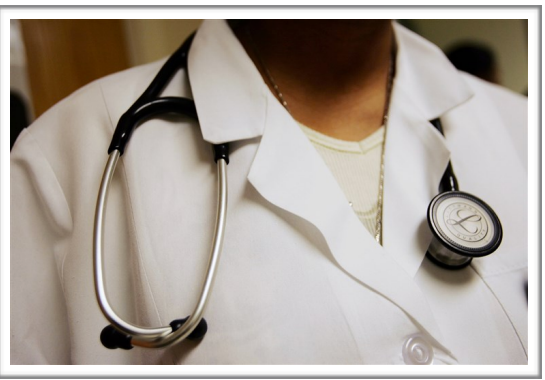

Source: (Joe Raedle/ Getty Images)

\section{Results}

Participants who reported

being less acculurated also reported:

- lower quality health care

- lower confidence filling out medical forms

- greater difficulty understanding written information about their medical conditions.

- greater discrimination in health care

\footnotetext{
${ }^{1}$ Becerra, D., Androff, D., Messing, J. T., Castillo, J., \& Cimino, A. (2015). Linguistic acculturation and perceptions of quality, access, and discrimination in health care among Latinos in the United States. Social work in health care, 54(2), 134-157.

${ }^{2}$ Linguistic acculturation consisted of three-item scale, one of the items asked, "would you prefer to be interviewed in English?" $(0=$ yes, $1=$ no). Participants were also asked (1) "Would you say you can carry on a conversation in English, both understanding and speaking ... "; and (2) "Would you say you can read a book, magazine, or newspaper in English ..." The response options were ( 1 = very well to $4=$ not at all).

${ }^{3}$ To measure Latinos' perceptions, participants were asked to rate the quality of medical care received in the past 12 months? $(1=$ poor to $4=$ excellent $)$. Access to medical information was measured with the following two items: (1) How confident are you filling out medical forms by yourself? $(1=$ not at all to $5=$ extremely); and (2) How often do you have problems learning about your medical condition because of difficulty understanding written information? $(1=$ never to $5=$ always).
}

Participants who identified having received poor quality medical treatment were asked additional follow-up questions about their perception of discrimination in regard to their medical care: Do you think you received poor quality of medical treatment or care because: (1) ... you were not able to pay?;... of your racial/ethnic background?; and (3) ... of your accent? The response options were $(0=$ no, $1=$ yes $)$. 


\section{Results}

Linguistic Acculturation and Perceptions of Care and Access to Health Information

The results of this study support all but one of the hypotheses. The findings indicate that there is a significant and negative association between linguistic acculturation and (I) quality of medical care, (2) confidence filling out medical forms, and a significant and positive association between linguistic acculturation and (3) difficulty understanding written information. In other words, participants with lower levels of linguistic acculturation were less likely to report high quality medical care or high confidence filling out medical forms. Also, participants with lower levels of acculturation were more likely to report greater difficulty understanding written information.

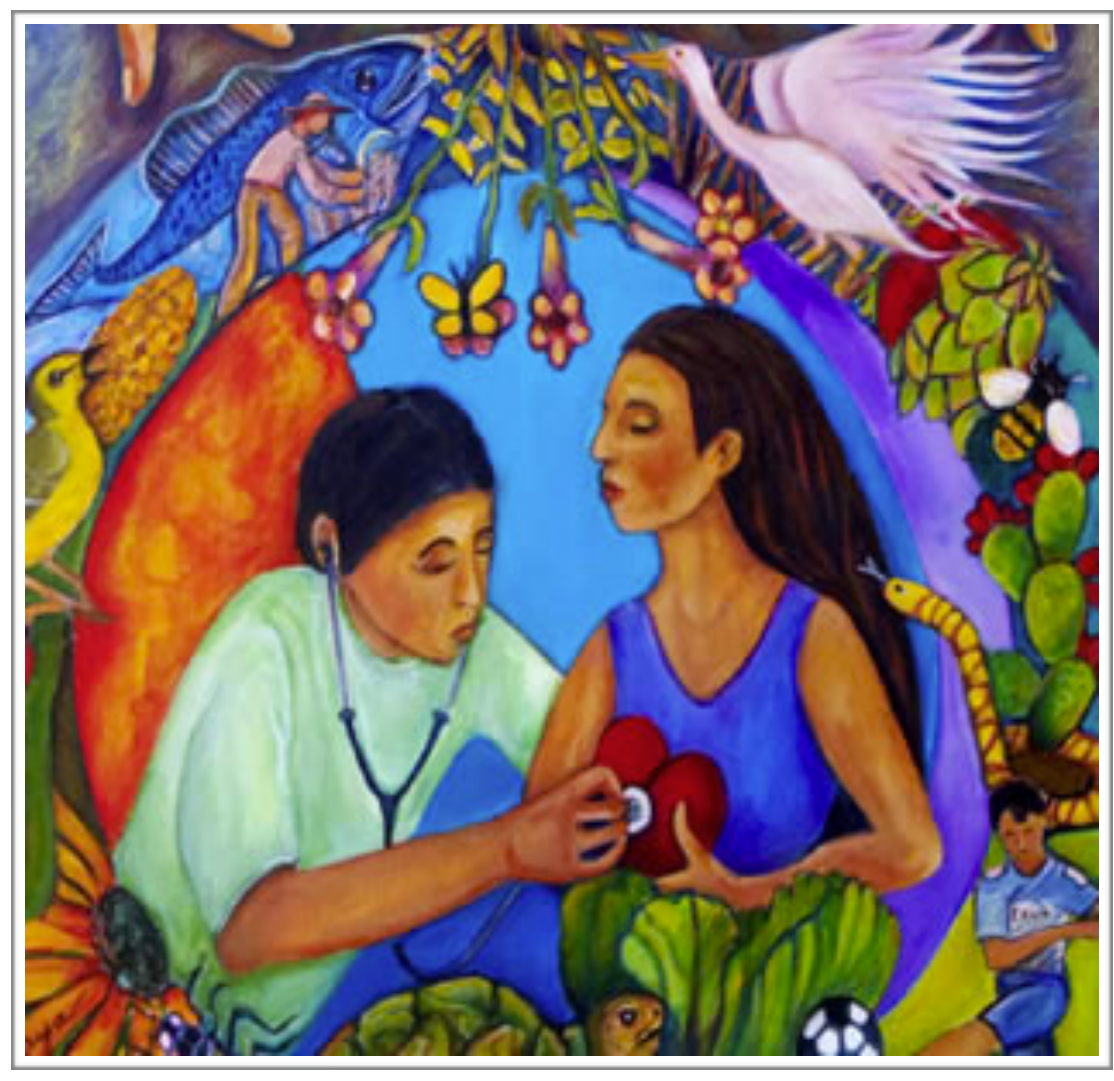

\section{Linguistic Acculturation and Perceptions of Poor Treatment and}

Source: (Maya Ramirez Schwarz)

\section{Discrimination in Medical Care}

The study also examined the relationship between acculturation and perceived discrimination in medical care amongst those who reported receiving poor quality medical treatment. Results indicated that immigrants were more likely than US-born participants to indicate that the poor quality of their treatment was due to their inability pay for services. Additionally, those with lower levels of linguistic acculturation were significantly more likely to indicate that their poor quality of care was a result of not being able to pay for services. Further, when compared to US-born participants, immigrants were three times more likely to indicate that poor quality of their medical treatment was the result of their race or ethnicity. Lastly, participants with lower levels of linguistic acculturation were more likely to report that their poor quality of care was a result of their accent.

\section{Conclusion}

This research supports the literature indicating that linguistic acculturation may restrict Latinos' access to quality health care treatment (Lara, Gamboa, Kahramanian, Morales, \& Hayes Bautista, 2005; Lillie-Blanton, Brodie, Rowland, Altman, \& McIntosh, 2000). Other studies have found that as the acculturation process progresses, the health of many Latino immigrants deteriorates (Aldrete et al., 2000; Torres, 2010). In this study, Becerra and colleagues (2015) found that that linguistic acculturation matters to perceptions of health care quality; more specifically, the results indicate that Latinos with lower levels of acculturation perceived (I) greater discrimination in health care treatment; (2) lower quality of health care treatment; (3) less confidence filling out medical forms; and $(4)$ greater challenge understanding written information about their medical conditions. The inability of less acculturated Latinos to understand and complete medical forms can lead to inaccurate diagnoses and/or prescriptions. The incorrect prescription of medications may cause interactions with other medications, overmedication, or trigger an allergic reaction. In addition, if less acculturated Latinos have difficulty understanding written medical forms, they may not adhere to treatment or misunderstand treatment instructions, which can have adverse consequences. For example, if a patient is instructed to take a medication "once daily," it can cause confusion to less linguistic acculturated Latinos because "once" translates to "eleven" in Spanish. Thus, it is necessary social workers and other health care professionals have an increased understanding of the complex health needs of less acculturated Latinos in order to advocate for culturally grounded interventions that overcome language barriers, which would positively impact the health care experience and treatment of less acculturated Latinos. 


\section{References}

Alderete, E., Vega, W.A., Kolody, B., Aguilar-Gaxiola, S. (2000). Lifetime prevalence of and risk factors for psychiatric disorders among Mexican migrant farmworkers in California. American fournal of Public Health, 90, 608-6r4.

Ayón, C., \& Becerra, D. (2013). Mexican immigrant families under siege: The impact of anti-immigrant policies, discrimination, and the economic crisis. Advances in Social Work, I4(I), 206-228.

Azuoni, M. (n.d.). [Digital Image]. https://www.theatlantic.com/health/archive/2OI4/o5/why-many-latinos-dread-going-to the-doctor/361547/

Cavazos-Rehg, P.A., Zayas, L.H., \& Spitznagel, E.L. (2007). Legal status, emotional well-being and subjective health status of Latino immigrants. Fournal of the National Medical Association, 99(Iо), II26-II3I.

Cheng, E.M., Chen, A., \& Cunningham, W. (2007). Primary language and receipt of recommended health care among Hispanics in the United States. Fournal of General Internal Medicine, 22(2), 283-288.

Cortado, X. (2003). [Digital Image]. "Salud"

http://umich.edu/_ac213/student_projectso7/latinahealth/imagesource.html\#salud

Ferguson, W.J., \& Candib, L.M. (2002). Culture, language, and the doctor-patient relationship. Family Medicine \& Community Health, 34(5), 353-36r.

Fiscella, K., Franks, P, Doescher, M., \& Saver, B. (2002). Disparities in health care by race, ethnicity, and language among the insured: Findings from a national sample. Medical Care, $40(\mathrm{I}), 5^{2-59}$.

Franzini, L., \& Fernandez-Esquer, M.E. (2004). Socioeconomic, cultural, and personal influences on health outcomes in low income Mexican-origin individuals in Texas. Social Science \& Medicine, 59(8), I629- I646.

Gonzalez, N., Fabrett, F. \& Knight, G. (2009). Acculturation, enculturation, and the psychological adaptation of Latino youth (pp. II5-134). In Villarruel, F., Carlo, G., Grau, J., Cabrera, N., Chahin, J. (eds.). Handbook of US Latino psychology: Developmental and community based perspectives. Thousand Oaks, CA. Sage Publications.

Huang, B., Appel, H., \& Ai, A.L. (2OII). The effects of discrimination and acculturation to service seeking satisfaction for Latina and Asian American women: Implications for mental health professions. Social Work in Public Health, 26(I), 46-59.

Humes, K.R., Jones, N.A., \& Ramirez, R.R. (201I). Overview of Race and Hispanic Origin: 2010 (U.S. Census Bureau Publication Number C2010BR-02). Washington, DC: U.S. Department of Commerce

Kouyoumdjian, H., Zamboanga, B.L., \& Hansen, D.H. (2003). Barriers to community mental health services for Latinos: Treatment considerations. American Psychological Association, ro (4), 394-422.

Lara, M., Gamboa, C., Kahramanian, M.I., Morales, L.S., \& Hayes Bautista, D.E. (2005). Acculturation and Latino health in the United States: A review of the literature and its sociopolitical context. Annual Review of Public Health, $26,267^{-} 297$.

Lillie-Blanton, M., Brodie, M., Rowland, D., Altman, D., \& McIntosh, M. (200o). Race, ethnicity and the health care system: Public perceptions and experiences. Medical Care Research and Reviere, 57(I), 218-235. 
Marsiglia, F.F., Kulis, S., Martinez-Rodriguez, G., Becerra, D., \& Castillo, J. (20o9). Culturally specific youth substance use resistance skills: Applicability across the U.S.-Mexico border. Research on Social Work Practice, 19(2), 152-164.

Peck, B.M., \& Denney, M. (20I2). Disparities in the conduct of the medical encounter: The effects of physician and patient race and gender. SAGE Open, $\mathrm{I}^{-}{ }^{-1} 4$. doi:IO.II77/21582440I2459193

Perez, D., Sribney, W.M., \& Rodriguez, M.A. (2009). Perceived discrimination and self-reported quality of care among Latinos in the United States. Fournal of General Internal Medicine, 24(3), 548-554.

Pérez-Escamilla, R. (201I). Acculturation, nutrition, and health disparities in Latinos. American fournal of Clinical Nutrition, 93, 163S-167S.

Perloff, R.M., Bonder, B., Ray, G.B., Ray, E.B., \& Siminoff, L.A. (2006). Doctorpatient communication, cultural competence, and minority health. American Behavioral Scientist, 49, 835-852.

Pilnick, A., \& Dingwall, R. (20II). On the remarkable persistence in asymmetry in doctor/patient interaction: A critical review. Social Science do Medicine, 72(8), I374-I382.

Pumariega, A.J., Rothe, E., \& Pumariega, J.B. (2005). Mental health of immigrants and refugees. Community Mental Health fournal, 4I, 58I-597.

Raedle, J. (n.d.). [Digital Image]. "A physician assistant wears a stethoscope.” https://www.nbcnews.com/news/latino/raul ruiz-only-latino-doctor-congress-troubled-gop-health-plan-n73605I

Ramirez-Schwarz, M. (n.d.). [Digital Image]. http://www.utsa.edu/today/20II/Io/healthforum.html

Sandars, J., \& Esmail, A. (2003). The frequency and nature of medical error in primary care: understanding the diversity across studies. Family practice, 20(3), 231-236.

Singh, H., Meyer, A.N.D., \& Thomas, E.J. (20I4). The frequency of diagnostic errors in outpatient care: Estimations from three large observation studies involving US adult populations. BMf 2uality \& Safety, online first. doi: 10.1136/bmjqs-2013-002627

Torres, L. (20IO). Predicting levels of Latino depression: Acculturation, acculturative stress, and coping. Cultural Diversity and Ethnic Minority Psychology, 16(2), 256-263.

Trivedi, A.M., \& Ayania, J.Z. (2006). Perceived discrimination and use of preventative health services. Fournal of General Internal Medicine, $21(6), 55^{-2} 55^{8}$.

Umaña-Taylor, A.J. \& Alfaro, E.C. (2009). Acculturative stress and adaptation. In Villarruel, F., Carlo, G., Grau, J., Cabrera, N., Chahin, J. (eds.). (pp. 135-252). Handbook of US Latino psychology: Developmental and community based perspectives. Thousand Oaks, CA. Sage Publications.

Vargas Bustamante, A., Fang, H., Rizo, J.A., \& Ortega, A.N. (2009). Understanding observed and unobserved health care access and utilization disparities among U.S. Latino adults. Medical Care Research and Review, 66(5), 56r 577 . 